\title{
Preparation of Diastereomeric $\beta$-Aryloxymethylaminoalcohols Containing Nicotinic Acid Moiety and Their Binding Affinity to $\boldsymbol{\beta}_{3}$-Adrenoreceptors
}

\author{
Seung Kyu Kang, Jae Du Ha, Haye-Gyeong Cheon, Joong-Kwoon Choi, Chang Sung Hong, and Eul Kgun Yum ${ }^{+, *}$ \\ Medicinal Science Division, Korea Research Institute of Chemical Technology, P.O. Box 107, Yaseong, Dayjeon 305-600, Korea \\ ${ }^{\dagger}$ Department of Chemistr, Chungnam National University; Yuseong, Daejeon 305-76-1, Korea \\ Received Ithe 17, 2003
}

Key Words : Diastereomer, $\beta-\Lambda$ minoalcohol, Nicotinic acid, $\beta_{s}$-Adrenoreceptors

The identification of the third $\beta$-adrenergic receptor subtype $\left(\beta_{\mathrm{Y}} \mathrm{AR}\right)$ led to the investigation of $\beta_{\text {s-adrenoreceptor }}$ agonists as potential agents for the treatment of various metabolic diseases.' Stimulation of $\beta$-adrenoreceptors on the surface of adipocytes evoked lipolysis and upregulation of the uncoupling protein (UCP1), which led to a net increase in energy utilization. ${ }^{2.3}$ Thus, $\beta_{\text {s-adrenoreceptor }}$ agonists may prove useful for the treatment of obesity. ${ }^{3}$ In addition, the agonists have also demonstrated a direct improvement on glucose tolerance for treatment of Type II (non-insulin dependent) diabetes. Recently, many pharmaceutical companies have developed $\beta_{s}$-adrenoreceptor agonists. which have shown highly selective binding affinity to $\beta_{\xi^{-}}$ adrenoreceptors (A-D). ${ }^{1}$ The literature reports have shown that the single diastereomer of $\beta_{\text {s }}$-adrenoreceptor agonists are often more potent or have less side effects compared to their racemates. ${ }^{5}$ Of the numberous methods for the preparation of chiral aryl substituted $\beta$-aninoalcohols, the most direct method is alkylation of the corresponding chiral anine with arylethylene oxide. ${ }^{6}$ However, direct alkylation in polar, protic solvents generally gave the desired products in low yields with significant amounts of regioisomer and multiply alkylated side products.

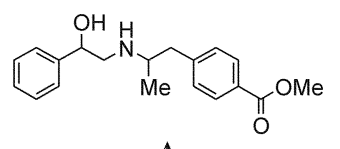

A

BRL 26,830<smiles>COCOc1ccc(OCNCC(O)COc2ccccc2)cc1</smiles>

C ICI 19,857

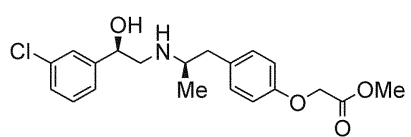

B

BRL 3,5135

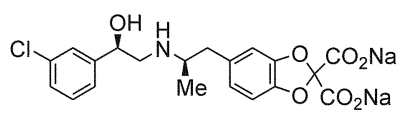

D

CL-316,243

Currently, heteroarylethanolamines have also been reported to show significant $\beta_{s}$ agonist activity and minimal cross-reactivity at the $\beta_{1}$ and $\beta_{2}$ receptors. ${ }^{8}$ The $\beta$-aminoalcohol could contain various heterocycles such as oxazole," pyridine, ${ }^{10}$ and indole. ${ }^{11}$ In an effort to discover new lead compounds for $\beta_{3}$-adrenoreceptor agonist, we were posed with the problem of finding efficient and direct route to

prepare optically pure diastereomeric $\beta$-arylaminoalcohols. We describe herein simple diastereomeric preparation of heterocyclic $\beta$-arylaminoalcohols containing nicotinic acid moiety and their binding affinity to $\beta$-adrenoreceptors.

\section{Chemistry}

The synthetic procedures for the preparation of diastereomeric $\beta$-aminoalcohols are detailed in Scheme 1. The (S)-1azido-3-phenoxypropane-3-ol (2) was obtained by the ring opening reaction of $(S)$-2-phenoxymethyloxirane (1) with $\mathrm{NaN}_{3}$ in $\mathrm{CH}_{3} \mathrm{CN}$ at $80{ }^{\circ} \mathrm{C}$. The hydrogenation of 1-azido-3phenoxypropane-2-ol (2) using $\mathrm{Pd} / \mathrm{C}$ provided 1-amino-3phenoxypropan-2-ol (3) in a cluantitative yield. The 5-(3oxobutyl)-nicotinic acid methyl ester (4) were prepared by palladium-catalyzed coupling reaction of 5-bromonicotinic acid methy] ester with 3-buten-2-ol in a 70-\% yield. ${ }^{12}$ The imino compound 5 was obtained by condensation of aminoalcohol 3 and ketone 4 by azeotrophic reflux in benzene. The diasteroisomeric mixture of $6 \mathbf{a}$ was prepared by lydrogenation of imine 5 with $\mathrm{PtO}_{2}$ catalyst under 60 psi hydrogen pressure in solvent. The Boc protected $\mathbf{6 c}$ and $\mathbf{6 d}$ were separated by MPLC with Merck l.obar RP-18 column and $\mathrm{CH}_{3} \mathrm{CN}: \mathrm{H}_{2} \mathrm{O}=1: 1$ as eluant. The compound $\mathbf{6 c}$ and 6d were obtained by deprotection of Boc group and neutralization. Another set of diastereomeric compounds $6 \mathbf{6}$ and $6 f$ were also prepared by the same procedure with Scheme 1 except for $(R)$-2-phenoxymethyl-oxirane as a chiral substrate (Scheme 2). The stereochemistry of $\mathbf{6 c - 6 f}$ were determined by comparison of literature spectra after ring formation to oxazolodinone with 1,1-carbonyldiimidazole..$^{13}$

\section{Screening Results}

lo determine the affinity of these $\beta$-aminoalcohols as $\beta_{\text {; }}$ adrenoreceptor agonists, the receptor binding assay was performed by using cell membrane expressing human $\beta_{\text {; }}$ adrenoreceptors (RB-HBETA $)_{3}{ }^{14}$ The data are summarized in lable 1. Unexpectedly, the heterocyclic aminoalcohols containing nicotinic ester have shown similar binding affinities except for $(R, S)$-isomer 6e which showed a quarter of the affinity compared to the other isomers. 


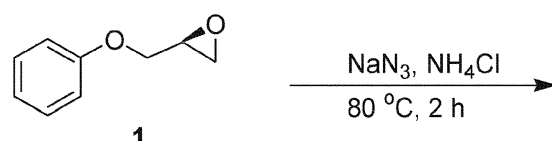<smiles>NCC(O)COc1ccccc1</smiles>

$\underset{\mathrm{MeOH}, \mathrm{rt}, 2 \mathrm{~h}}{\stackrel{5 \% \mathrm{Pd} / \mathrm{C}, 50 \mathrm{Psi} \mathrm{H}}{2}}$<smiles>NCC(O)COc1ccccc1</smiles><smiles>COC(=O)c1cncc(CCC(C)=O)c1</smiles>
bezene, refulx<smiles>COC(=O)c1cncc(CC/C(C)=N/C[C@H](O)COc2ccccc2)c1</smiles><smiles>COC(=O)c1cncc(CCC(C)NC[C@H](O)COc2ccccc2)c1</smiles>

$6 a$

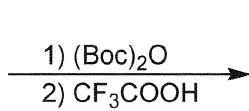<smiles>COC(=O)c1cncc(CC[C@H](C)NC[C@H](O)COc2ccccc2)c1</smiles>

$6 c$<smiles>COC(=O)c1cncc(CC[C@H](C)NC[C@H](O)COc2ccccc2)c1</smiles>

$6 d$<smiles>COC(=O)c1cncc(CCC(C)NC[C@H](O)COc2ccccc2)c1</smiles>

Scheme 2
Table 1. Comparison of the $\beta_{\mathrm{i}}$ AR Affinity of Diasteremeric $\beta$ Aminoalcohols

\begin{tabular}{ccccc}
\hline Entry & Compound & Configuration & $\mathrm{IC}_{511}(\mu \mathrm{M})$ & $\mathrm{Ki}(\mu \mathrm{M})$ \\
\hline $\mathrm{I}$ & $\mathbf{6 c}$ & $S_{S} S$ & 1.28 & 0.67 \\
2 & $\mathbf{6 c}$ & $S_{S} R$ & 1.15 & 0.61 \\
3 & $\mathbf{6 e}$ & $R_{S} S$ & 4.57 & 2.41 \\
4 & $6 \mathrm{f}$ & $R_{.} R$ & 1.10 & 0.58 \\
5 & BRL-35135 & $S . S$ & 3.62 & 1.91 \\
6 & C.L-316243 & $S . S$ & 1.17 & 0.62 \\
\hline
\end{tabular}

\section{Conclusions}

The four diastereomers of heterocyclic $\beta$-aminoalcohols were easily prepared by separation of their Boc derivatives as the key step. The introduction of nicotinic acid moiety to $\beta$-aminoalcohols resulted in potent $\beta_{5}$-adrenergic receptor binding affinity. The nicotinic acid moiety could be a potential heterocyclic substrate for the development of $\beta_{3}$ adrenoreceptor agonists.

\section{Experimental Sections}

All chemicals were purchased and used without any further purifications The ' $\mathrm{H}$ NMR spectra were obtained on a Varian Gemini $200 \mathrm{MHz}$ or Bruker $300 \mathrm{MHz}$ NMR Spectrometer. The GC-MS spectral were obtained on a Shimazu QP 1000 mass spectrometer. Melting points were deterimined on MU-TEM apparatus and were uncorrected. BRL-35I35 and CL-316243 were prepared literature procedures $^{\text {th }}$ and used as reference compounds.

(S)-2-Phenoxymethyloxirane (1) ${ }^{15}$. $\mathrm{NaH}(60 \%$ dispersion in inineral oil, $0.72 \mathrm{~g}, 18 \mathrm{mmol}$ ) was added to a solution of phenol ( $1.23 \mathrm{~g} .13 \mathrm{minol})$ in dry DMF $(10 \mathrm{~mL})$ and the resulting suspension was stirred for approximately 30 minutes until a clear solution was obtained. A solution of $(S)$-(-)-glycidyl 3-nitrobenzenesulfonate $(3.1 \mathrm{g.} .12 \mathrm{mmol})$ in dry DMF (7 $\mathrm{mL}$ ) was slowly added to phenoxide solution. The inixture was stirred for 6 hours at $20^{\circ} \mathrm{C}$ and poured into saturated aqueous $\mathrm{NH}_{1} \mathrm{Cl}$ solution $(50 \mathrm{~mL})$. The product was extracted with ethyl ether $(3 \times 20 \mathrm{~mL})$. The ethyl ether layer was dried over anhydrous $\mathrm{MgSO}$, and concentrated. The $(S)$ 2-phenoxymethyl oxirane was obtained $86 \%$ yields by silica gel column chromatography.

${ }^{\prime} \mathrm{H}$ NMR (CDCl, $\left.300 \mathrm{MHz}\right) \delta 7.30-7.24(\mathrm{~m}, 2 \mathrm{H}), 6.98-$ $6.89(\mathrm{~m}, 3 \mathrm{H}), 4.19(\mathrm{dd}, J-10.9,3.2 \mathrm{~Hz}, \mathrm{IH}), 3.93\left(\mathrm{dd}, J^{-}\right.$ $11.3,5.6 \mathrm{~Hz}, \mathrm{lH}), 3.33(\mathrm{~m}, 1 \mathrm{H}), 2.87$ (t. $J-4.9 \mathrm{~Hz}, \mathrm{IH})$, $2.73(\mathrm{dd}, J-4.9,2.6 \mathrm{~Hz}, \mathrm{IH})$; Mass $\mathrm{m} / \mathrm{e}(\%) 150(\mathrm{M}, 26)$, $119(10), 107(35), 94(100), 77(50), 65(40)$.

(S)-1-Azido-3-phenoxypropane-2-ol (2). The mixture of $0.6 \mathrm{~g}(5 \mathrm{mmol})$ of (S)-2-phenoxymethyloxirane (1), $1.52 \mathrm{~g}$ (25 mmol) of $\mathrm{NaN}_{3}$, and $\mathrm{H}_{2} \mathrm{O}$-acetonitrile $(1: 8,9 \mathrm{~mL})$ in 25 $\mathrm{mL}$ flask was stirred at $80^{\circ} \mathrm{C}$ for 4 hours. The mixture was poured into $20 \mathrm{~mL}$ of cold water. The product was extracted with ethyl ether $(2 \times 20 \mathrm{~mL})$. The organic layer was washed saturated $\mathrm{NH}_{4} \mathrm{Cl}$ solution $(20 \mathrm{~mL})$ and water. The ethyl ether layer was dried over anhydrous $\mathrm{MgSO}_{4}$ and concentrated. The $(S)$-1-azido-3-phenoxypropane-2-ol was obtained $97 \%$ yields by silica gel column chromatography.

${ }^{\prime} \mathrm{H}$ NMR (CDCl $\left.300 \mathrm{MHz}\right) \delta 7.31-7.24(\mathrm{~m}, 2 \mathrm{H}), 7.00-$ $6.88(\mathrm{~m}, 3 \mathrm{H}), 4.16(\mathrm{~m}, \mathrm{lH}), 3.93(\mathrm{~d}, J-5.6 \mathrm{~Hz}, \mathrm{lH}), 3.50$ (m, IH), 2.7। (brs, lH); Mass in/e (\%) I67 (M , 3), I49(4), 123 (23), 94 (100), 77 (25).

(S)-1-Amino-3-phenoxypropan-2-ol (3). The mixture of 
(S)-1-azidophenoxypropane-2-ol (1.71 g. $8.9 \mathrm{mmol}$ ) and $5 \%$ $\mathrm{Pd} / \mathrm{C}(0.2 \mathrm{~g})$ and methanol $(15 \mathrm{~mL})$ in pressure bottle was hydrogenated under $60 \mathrm{psi}$ of hydrogen for $4 \mathrm{~h}$ at room temperature. The resulting solution was filtered and concentrated. The was obtained $88 \%$ yields by silica gel colunin chronatography. mp $104-106{ }^{\circ} \mathrm{C}$ : ${ }^{1} \mathrm{H} \mathrm{NMR} \mathrm{(CDCl}{ }_{3}$. $300 \mathrm{MHz}) \delta 7.31-7.26(\mathrm{~m}, 2 \mathrm{H}), 6.98-6.90(\mathrm{~m}, 3 \mathrm{H}), 4.01-$ $3.91(\mathrm{~m}, 3 \mathrm{H}) .2 .98(\mathrm{dd}, J=12.8,3.7 \mathrm{~Hz} . \mathrm{lH}), 2.86(\mathrm{dd} . J=$ $12.8,6.4 \mathrm{~Hz} . \mathrm{IH})$; Mass m/e (\%) $193\left(9, \mathrm{M}^{-}\right) .119(34) .107$ (21), $94(100), 77(65) .65(26)$.

5-(3-0xobutyl)nicotinic acid methyl ester (4). To a 10$\mathrm{mL}$ vial containing a magnetic stirring bar was added the following reagents: $\mathrm{Pd}(\mathrm{OAc})=(0.025 \mathrm{mmol}), \mathrm{KOAc}(1.0$ munol), $\mathrm{LiCl}(0.5 \mathrm{mmol})$, 3-buten-2-ol (1.0 mmol), methyl 5 bromonicotinate $(0.5 \mathrm{mmol})$ and $\mathrm{DMF}(5 \mathrm{~mL})$. The vial was sealed with a septum. The mixture was stirred at the $110^{\circ} \mathrm{C}$ for 4 hours. The resulting nixture was diluted with ethyl acetate $(20 \mathrm{~mL})$ and washed with saturated aqueous $\mathrm{NH}_{4} \mathrm{Cl}$ $(2 \times 20 \mathrm{~mL})$. The ethyl acetate layer was dried over anhydrous $\mathrm{MgSO}_{4}$ and concentrated. The product was obtained $70 \%$ yields by flash colunu chromatography. mp: $53.54{ }^{\circ} \mathrm{C}$ : ${ }^{1} \mathrm{H} \mathrm{NMR}\left(\mathrm{CDCl}_{3}, 300 \mathrm{MHz}\right) \delta 8.96(\mathrm{~d}, \mathrm{lH} . J=2.0$ Hz). 8.66 (t. $1 \mathrm{H}, J=2.4 \mathrm{~Hz}$ ). 8.05 (d, lH. $J=2.0 \mathrm{~Hz}$ ). 3.87 (s. $3 \mathrm{H}), 2.89$ (t. $2 \mathrm{H} . J=7.4 \mathrm{~Hz}), 2.76$ (t. $2 \mathrm{H} . J=7.2 \mathrm{~Hz}$ ). 2.09 (s. $3 \mathrm{H})$ : Mass n/e (\%) $207\left(13 . \mathrm{M}^{-}\right) .164(75), 150$ (14). 132 (32). $104(24) .77(14), 43(100)$.

5-[3-(2-Hydroxy-3-phenoxypropylamino)butyl]nicotinic acid methyl ester (6a). A mixture of (S)-1-amino-3phenoxypropan-2-ol (3) (1.0 nmol), 5-(3-oxobutyl)nicotinic acid methyl ester (4) (1.0 mumol). molecular sieve (2 g) and benzene $(20 \mathrm{~mL})$ in $50 \mathrm{~mL}$ flask was heated under azeotropic reflux for 20 hours. The resulting solution was filtered and concentrated. The 5-[2-(2-hydroxy-3-phenoxypropylimino)propyl]nicotiuic acid methyl esters (5) was obtained $80 \%$ yields as oil. The crude inine (5) and $\mathrm{PtO}_{2}(50$ $\mathrm{mg}$ ) were added to methanol $(15 \mathrm{~mL})$ in pressure bottle. The mixture was hydrogenated under $70 \mathrm{psi}$ hydrogen for $4 \mathrm{~h}$ at roon temperature. The resulting solution was filtered and concentrated. The aminoalcohol (6a) was obtained $63 \%$ yields by silica gel column chromatographỵ. ${ }^{1} \mathrm{H}$ NMR $\left(\mathrm{CDCl}_{3}, 200 \mathrm{MHz}\right) \delta 9.02(\mathrm{~d} .1 \mathrm{H} . J=2.0 \mathrm{~Hz}) .8 .60$ (t. $1 \mathrm{H} . J$ $=2.0 \mathrm{~Hz}$ ). 8.11 (d. $1 \mathrm{H} . J=2.4 \mathrm{~Hz}) .7 .24$ (t. $2 \mathrm{H} . J=8.0 \mathrm{~Hz})$. $6.89-6.87(\mathrm{~m} .3 \mathrm{H}) .4 .15-3.91(\mathrm{~m} .3 \mathrm{H}) .3 .88(\mathrm{~s} .3 \mathrm{H}) .3 .63 \mathrm{~m}$. 2H), 2.89-2.70 (m, 5H), 1.86-1.67 (m. 2H), 1.19-1.16 (m. $3 \mathrm{H})$ : Mass m/e (\%) $359\left(100 . \mathrm{M}^{-1}\right) .332(12) .181(6) .149$ (12). $111(13) .96(14) .68(13), 55(12), 44(37)$.

Separation of Boc protected $6 \mathrm{c}$ and $6 \mathrm{~d}$. 5-[3-(2-Hydroxy-3-phenoxypropylamino)butyl]nicotinic acid methyl ester (6a. mmol) and (Boc) $)_{2} \mathrm{O}$ were dissolved in $20 \mathrm{~mL}$ of $\mathrm{CH}_{2} \mathrm{Cl}_{2}$. The reaction mixture was stirred about $12 \mathrm{~h}$ at room temperature. The Boc protected 6 a was obtained quantitatively by concentration. The Boc protected diastereomers of 6c and 6d were separated by MPLC with Merck Lobar RP18 column $\left(440 \times 37 \mathrm{~mm}\right.$. \#10626) and $\mathrm{CH}_{3} \mathrm{CN}: \mathrm{H}_{2} \mathrm{O}=$ $1: 1$ eluent (UV-254 $\mathrm{nM}$ and $10 \mathrm{~mL} / \mathrm{min}$ ). The diastereoselectivity of $\mathbf{6 c}$ and $6 \mathbf{d}(44: 56)$ was determined by HPLC with Waters Spherisor S 10 ODS2 $(250 \times 4.6 \mathrm{~mm}$.
\#PS832515) and $\mathrm{CH}_{2} \mathrm{CN}: \mathrm{H}_{2} \mathrm{O}=1: 1$ eluent (UV-254 nM and $1.0 \mathrm{~mL} / \mathrm{min}$ ). Boc-protected $6 \mathrm{c}:{ }^{1} \mathrm{H}$ NMR (CDCl 2.200 $\mathrm{MHz}) \delta 9.05(\mathrm{~d}, J=2.0 \mathrm{~Hz} . \mathrm{HH}) .8 .60(\mathrm{~d}, \mathrm{lH}, J=2.2 \mathrm{~Hz})$, 8.10 (t. $J=2.0 \mathrm{~Hz}, \mathrm{lH}$ ), $7.30-7.22$ (m. $2 \mathrm{H}$ ), $6.90-6.85$ (m. $3 \mathrm{H}$ ). 4.90 (brs. lH), 4.13-4.02 (m. $4 \mathrm{H}$ ). 3.91 (s. $3 \mathrm{H}), 3.42$ (brs. $2 \mathrm{H}), 2.67$ (t. $J=7.9 \mathrm{~Hz}, 2 \mathrm{H}) .1 .94(\mathrm{~m} . \mathrm{lH}), 1.77(\mathrm{~m}$, 2H). 1.47 (s. 9H). 1.21 (d. $3 \mathrm{H} . J=6.4 \mathrm{~Hz}$ ). Boc-protected 6d: ${ }^{1} \mathrm{H}$ NMR $\left(\mathrm{CDCl}_{3}, 200 \mathrm{MHz}\right) \delta 9.06($ d. $J=1.8 \mathrm{~Hz} . \mathrm{lH})$, 8.58 (d. $1 \mathrm{H}, J=1.8 \mathrm{~Hz}) .8 .10(\mathrm{t} . J=1.8 \mathrm{~Hz} .1 \mathrm{H}), 7.26$ (t. $2 \mathrm{H}$, $J=6.8 \mathrm{~Hz}) .6 .90(\mathrm{~m}, 3 \mathrm{H}) .4 .90(\mathrm{brs}, \mathrm{HH}) .4 .20-3.9 \mathrm{l}(\mathrm{m} .4 \mathrm{H})$, $3.91(\mathrm{~s}, 3 \mathrm{H}), 3.42(\mathrm{brs}, 2 \mathrm{H}), 2.67(\mathrm{t} . J=7.9 \mathrm{~Hz}, 2 \mathrm{H}), 1.94(\mathrm{~m}$, lH). $1.77(\mathrm{~m}, 2 \mathrm{H}), 1.47(\mathrm{~s} .9 \mathrm{H}), 1.2 \mathrm{l}(\mathrm{d}, 3 \mathrm{H} . J=6.4 \mathrm{~Hz})$.

(S,S)-5-[3-(2-Hydroxy-3-phenoxy]propylamino)butyl]nicotinic acid methyl ester $(6 \mathrm{c})$. The Boc-protected $6 \mathrm{c}$ (1 mmol) was dissolved in $\mathrm{CH}_{2} \mathrm{Cl}_{2}(10 \mathrm{~mL})$. The trifluoroacetic acid (5 eqiuv) was added to the solution. The reaction mixture was stirred for $12 \mathrm{~h}$ at room temperature and neutralized with saturated $\mathrm{Na}_{2} \mathrm{CO}_{3}$ solution. The organic layer was separated and concentrated. The compound $6 \mathrm{c}$ was obtained $85 \%$ yields as oil by silica gel column chromatography. ${ }^{~} \mathrm{H} \mathrm{NMR}\left(\mathrm{CDCl}_{3,}, 200 \mathrm{MHz}\right) \delta 8.96$ (d. $J=1.8 \mathrm{~Hz}$, lH). $8.53(\mathrm{~d}, \mathrm{lH} . J=1.8 \mathrm{~Hz}) .8 .04$ (t. $J=1.8 \mathrm{~Hz} .1 \mathrm{H}), 7.26$ (t. $2 \mathrm{H} . J=6.8 \mathrm{~Hz}), 6.85(\mathrm{~m} .3 \mathrm{H}), 4.0 \mathrm{l}-3.89(\mathrm{~m}, 3 \mathrm{H}) .3 .86(\mathrm{~s}$, $3 \mathrm{H}) .2 .85-2.62(\mathrm{~m} .7 \mathrm{H}) .1 .67(\mathrm{~m} .2 \mathrm{H}) .1 .47(\mathrm{~s}, 9 \mathrm{H}), 1.08(\mathrm{~d}, J$ $=6.3 \mathrm{~Hz} .3 \mathrm{H})$ : Mass $(m e) 358\left(8, \mathrm{M}^{+}\right) .221(\mathrm{l} 00), 194(27)$.

(S,R)-5-[3-(2-Hydroxy-3-phenoxypropylamino)butyl]nicotinic acid methyl ester (6d). ${ }^{j} \mathrm{H}$ NMR $\left(\mathrm{CDCl}_{3}, 200\right.$ $\mathrm{MHz}) \delta 8.97(\mathrm{~d}, J=2.0 \mathrm{~Hz} .1 \mathrm{H}) .8 .54(\mathrm{~d}, 1 \mathrm{H}, J=2.0 \mathrm{~Hz}$ ), 8.04 (t. $J=2.2 \mathrm{~Hz}, 1 \mathrm{H}$ ), 7.24-7.16 (m. 2H), 6.91-6.80 (m. $3 \mathrm{H}$ ). 4.03-3.83 (m 3H). 3.87 (s. 3H), 2.97-2.60 (m. 7H), 1.68 (m. $2 \mathrm{H}), 1.08$ (d, $J=6.3 \mathrm{~Hz} .3 \mathrm{H}$ ): Mass $(m e) 358\left(5.6 . \mathrm{M}^{-}\right)$. $221(100) .194(29)$.

(R,S)-5-[3-(2-Hydroxy-3-phenoxypropylamino)butyl]nicotinic acid methyl ester (6e). ${ }^{j} \mathrm{H}$ NMR (CDCl, 200 $\mathrm{MHz}) \delta 8.97$ (d. $J=2.0 \mathrm{~Hz} . \mathrm{lH}$ ) 8.54 (d. $1 \mathrm{H}, J=2.0 \mathrm{~Hz}$ ), 8.04 (t. $J=2.2 \mathrm{~Hz}, 1 \mathrm{H}$ ), 7.24-7.16 (m. 2H), 6.91-6.80 (m. $3 \mathrm{H}$ ). 4.03-3.83 (m, 3H). 3.87 (s. $3 \mathrm{H}), 2.97-2.60$ (m. $7 \mathrm{H})$, 1.68 (m. $2 \mathrm{H}) .1 .08$ (d. $J=6.3 \mathrm{~Hz} .3 \mathrm{H})$; Mass $(\mathrm{m} / \mathrm{e}) 359(70$, $\left.\mathrm{M}^{-1}\right) .221(100) .194(30.1)$.

$(R, R)-5-[3-(2-H y d r o x y-3-p h e n o x y p r o p y l a m i n o) b u t y]]-$ nicotinic acid methyl ester (6f). ${ }^{1} \mathrm{H}$ NMR $\left(\mathrm{CDCl}_{3} .200\right.$ $\mathrm{MHz}) \delta 8.96$ (d. $J=1.8 \mathrm{~Hz}, 1 \mathrm{H}) .8 .53(\mathrm{~d} .1 \mathrm{H}, J=1.8 \mathrm{~Hz}$ ). 8.04 (t. $J=1.8 \mathrm{~Hz}, 1 \mathrm{H}) .7 .26(\mathrm{t} .2 \mathrm{H} . J=6.8 \mathrm{~Hz}), 6.85(\mathrm{~m}$. $3 \mathrm{H}) .401-3.89$ (m. 3H). 3.86 (s. 3H), 2.85-2.62 (m. 7H). 1.67 (m. 2H). 1.47 (s. 9H). 1.08 (d. $J=6.3 \mathrm{~Hz}, 3 \mathrm{H})$ : MS $(m e), 359\left(68.0 . \mathrm{M}^{-1}\right) .221(100) .194(25.3)$

Measurement of $\boldsymbol{\beta}$-adrenoceptor binding affinity. To determine the binding affinity of $\mathbf{6 c - 6}$ of on $\beta_{2}$-adrenorecetor. RB-HBETA3 membrane was incubated with [ $\left.{ }^{125} \mathrm{I}\right]-$ iodocyanopindolol (1.4 nM. $2200 \mathrm{Ci} / \mathrm{mmol})$ and unlabeled ligand for $10 \mathrm{~min}$ at $37^{\circ} \mathrm{C}$. Propranolol $(1 \mathrm{mM})$ was used to define non-specific binding. Incubation mixture was filtered over glass fiber (Wallac 140-521). washed and measured for radioactivity.

Acknowledgments. This work was supported by Ministry of Science and Technology and Bioneer Corporation. 


\section{References}

1. Arch. J. R. S.: Kaumantn. A. I. Medcinal Resarch Resiew 1993. 13. 663.

2. (a) Arch. I. R: Ainsworth. A. T: Canthome. M. A.: Piercy, V: Sennitt. M. V: Thody, V. E: Wilson, C: Wilson, S. Nature 1984. 309. 163. (b) Lowell. B. B.: Filer I. S. Amm. Rev. Hed 1997, 48. 307. (c) Strosberg. A. D.: Pietri-Rouxel. F. Trends Pharmacol. Soc $1996.206,373$

3. Arch. J. R. S.: Wilson. S. Int J. Obesitt 1996. 20.191.

4. (a) Claus, T. H.: Bloom. I. D. Annal Reports in Medicinal Chemisty 1995, 30, 189. (b) Howe, R. Drug of the Future 1993. 18,529

5. Devocelle. M.: Morteux. A.: Agbossou. F.: Dormoy. J.R. Tetrathedron Lett. 1999, 40.4551 and references therein.

6. Hett. R.: Fang. Q. K.: Gao. Y: Hong. Y.: Butler. H. T: Nie. X.: Wald. S. A. Tetrahedon Lett. 1997.38.1125 and references therein.

7. Atkins, R. K, Frazier, I.: Moore, L. L.; Weigel. L. O. Tetrahedron Lett. 1986. 27. 2451.

8. Mathink. R. J.: Tolman. S. M.: Chitty. D.: Candelore. M. R.: Cascieri. M. A.: Colwell. L. F.: Deng. J. L.: Feeney. W. P.: Forrest. M. J.: Hom. G. J.: MacIntyre. D. E.: Miller. R. R.: Stearns. P. A.: Tota, L.: Wyvratt, M. I.: Fisher. M. H.: Weber A. E. J. Med. Chem. 2000 - 13. 3832

9. Biftu. T.: Feng. D. D.: Ling G. B.: Kuo. H.: Qina. X.: Naylor. E. M.: Colandrea. V. J.: Candelore. M. R.: Casieri. M. A.: Colwell. L. F.: Forrest. M. J.: Hom. G. J: MacIntyre. D. E.: Miller. R. R.: Stearns. P. A.: Tota. L.: Wyvratt. M. J.: Fisher. M. H.: Weber. A. E. Bioorg. Afed Chent Lett. 2000. 10. 1431.

10. (a) Ok: H. O: Reigle. L. B. Candelore. M. A: Colwell, L. F: Deng, L.; Feener; W. P. F; Forrest, M. I.: Hom. G. I.: Macintyre, D. E.: Strader. C. D.: Tota. L.: Wang. M. J.: Wyvratt. M. J.: Fisher. M. H.: Weber. A. E. Bioorg. Med Chem. Lett 2000. 10. 1531. (b)
Shih. T. L.: Candelore. M. R: Cascieri, M. A.: Chiu. S. L: Colwell. L. F.: Deng. J. L.: Feeney. W. P.: Forrest. M. J.: Hom. G. J: Macintyre. D. E: Miller. R. R.: Stearns. P. A.: Tota. L. Wyratt. M. J.: Fisher. M. H.: Weber. A. E. Bioorg Med. Chent. Lett. 1999, 9, 1251. (c) Naylor, E. M.; Parmee, E. R.; Colandrea, V. J.: Perkins. L.; Brockunier, L.: Candelore. M. R.; Cascieri, M. A.; Colwell. L. F; Mathvink. R. J.: Deng, J. L.; Feeney, W. P. Forrest. M. T.: Hom. G. T.: MacIntyre. D. E.: Strader. C. D.: Tota. L.: Wang. P.-R.: Wyvratt. M. J.: Fisher. M. H.: Weber. A. E. Bioorg. Med Chent Lett 1999. 9. 755. (d) Parmee. E. R.: Naylor. E. M.: Perkins. L.; Colandrea. V. J.: Ok, H. O : Colandrea, V. J.; Cascier, M. A.; Deng. J. L.: Feeney, W. P: Forrest. M. J.; Hom, G. J; Macintyre. D. E; Miller. R. R:- Stearns. R. A.; Strader. C. D.: Tota. L.: Wang. P.-R.: Wyvratt. M. J.: Fisher. M. H.: Weber. A. E. Bioorg. Hed Chent Lett 1999. 9.749.

11. Mathvink. R. J.: Barritta. A. M.: Candelore. M. R.: Cascieri. M. A.; Deng. L.: Tota, L.; Strader, C. D.; Wyvratt, M. J:- Fisher. M H. Weber, A. E. Bioong. Med Chem Lett 1999. 9. 1869.

12. Yum. E. K.: Kang. S. K: Choi, J.-K. Bull. Konam Chem Soc 2001. 22. 644

13. Sher. P. M.: Plainsboro. N. T. 1996, US5.488.064.

14. Fisher. M. H.: Amend. A. M.: Bach. T. I.: Baker. T. M.: Brady. E J.; Candelore. M. R.; Carroll. D.: Cascieri, M. A.: Chiu. S.-H. L. Deng. L.: Forrest, M. J.: Hegarty-Friscino, B.; Guan, X.-M.: Hom. G. J.: Hutchins, J. E.: Kelly. L. J.: Mathvik. R. J:; Metzger, J. M. Miller. R. R.: Ok. H. O.: Parmee. E. R.: Saperstein. R.: Strader. C. D.: Steams. R. A.: Thompson. G. M.: Tota. L.: Vicario. P. P. Weber. A. E: Woods. T. W: Wyvratt. M. T.: Zafian. P. T: Macintyre, D. E. J. Clim Inest 1998, 101. 2387.

15. (a) McClure, D. E.: Arison, B. H.: Baldwin, J. J. J. Am Chem Soc. 1979. 101, 3666. (b) Klunder. J. M.: Onami. T.; Sharples. K B. J. Org Chem 1989. 54. 1295. (c) Fisher. M. H.: Parmee. E. R.: Mathvink. R. T.: Weber. A. E.: Ok. H. O. 1994. EP 0611003Al. 